Seguridad Operacional y Logística Aeronáutica

Segurança Operacional e Logística Aeronáutica

Operational Safety and Aviation Logistics

\title{
IMPLEMENTACIÓN DE TECNOLOGÍA AERONÁUTICA PARA APAGAR INCENDIOS FORESTALES EN EL TERRITORIO COLOMBIANO ${ }^{1}$
}

IMPLEMENTAÇÃO DA TECNOLOGIA AERONÁUTICA PARA APAGAR INCENDIOS NAS FLORESTAS NO TERRITÓRIO COLOMBIANO ${ }^{2}$ IMPLEMENTATION OF AERONAUTICAL TECHNOLOGY TO EXTINGUISH FOREST FIRES IN COLOMBIAN TERRITORY ${ }^{3}$

Mayra Mestizo Sosa ${ }^{4}$

Escuela Superior de Guerra

\section{CIENCIA Y PODER AÉREO}

ISSN 1909-7050 / E- ISSN 2389-2468 / Volumen 11/ Enero-diciembre de 2016/ Colombia/ Pp. 58-65 Recibido: 13/08/2016

Aprobado evaluador interno: 30/08/2016

Aprobado evaluador externo: 28/09/2016

Doi: http://dx.doi.org/10.18667/cienciaypoderaereo.514 
Resumen: este artículo presenta la implementación de tecnologías aeronáuticas para atender la necesidad inmediata que implican los cambios climáticos planteando la urgencia en la adquisición de una flota de aviones para apagar incendios forestales. Para esto, el Estado colombiano debe asegurar la implementación de tecnologías que permitan aplicar una solución dinámica según las contingencias detectadas, en un panorama de razonable manejo del riesgo, con un apoyo eficaz en la cobertura del territorio nacional, a bomberos de Colombia ante situaciones críticas y de peligro. Las evidencias objetivas, situaciones y datos presentados, permitirán comprender la necesidad inmediata a satisfacer por parte del Estado, en la búsqueda permanente de una nación más humana.

Palabras clave: Air Tractor AT-802; reforestación y restauración; desastre agroforestal; incendio forestal; riesgo ambiental.

${ }^{7}$ Artículo científico original, derivado del ejercicio investigativo del proyecto "Protección de los recursos vegetacionales del territorio nacional colombiano", presentado al banco de proyectos del Banco de la República. Bogotá, Colombia.

${ }^{2}$ Artigo científico original derivado do exercício investigativo do projeto "Proteção dos recursos vegetais do território nacional colombiano", apresentado para o banco de projetos do Banco de la República Bogotá, Colômbia.

${ }^{3}$ Original scientific article derived from the investigative exercise of the project "Protection of vegetation resources of the Colombian national territory, presented to the project bank of the Banco de la República. Bogota, Colombia.

${ }^{4}$ Ingeniera Industrial egresada de la Universidad Autónoma de Colombia, Magíster en Derechos Humanos y Derecho Internacional del Conflicto Armado por la Escuela Superior de Guerra de Colombia. Especialista en Producción Industrial, Control Fiscal y Ambiental, Estándares Internacionales y Aseguramiento Metrológico Industrial. Instructor en Empresas del Estado de primer nivel. Investigador en Derecho Internacional de los Derechos Humanos, Consultant in International Public Management Standards. Colombia. Correo electrónico: mmayramestizo@yahoo.com
Resumo: este artigo apresenta a implementação de tecnologias aeronáuticas para atender a imediata necessidade que significam as mudanças climáticas informando a urgente necessidade de adquirir um grupo de aviões para apagar incêndios florestais. Para isto, o Estado Colombiano deve garantir a implementação de tecnologias que permitam aplicar uma solução dinâmica consequentemente com as contingências encontradas, num panorama de manejo razoável do risco, com um apoio eficaz na cobertura do território nacional, com os bombeiros da Colômbia perante situações críticas e de perigo. As evidencias objetivas, situações e dados apresentados, vão permitir compreender a imediata necessidade de satisfazer, por parte de Estado, na procura permanente duma nação mais humana.

Palavras-chave: Air Tractor AT-802; reflorestamento e restauração; desastre agro-florestal; incêndio florestal; risco ambiental.

Abstract: This article presents the implementation of aeronautical technologies to meet the immediate need that the climate changes raising the urgency in the acquisition of a fleet of airplanes to extinguish forest fires. For this, the Colombian State must ensure the implementation of technologies that allow the application of a dynamic solution according to the detected contingencies, in a scenario of reasonable risk management, with effective support in the coverage of the national territory, to Colombian Firemen in critical and dangerous situations. The objective evidences, situations and data presented will allow us to understand the immediate need to be met by the State, in the permanent search for a more humane nation.

Key Words: Agroforestry disaster; Air Tractor AT-802; environmental risk; forest fire; Reforestation and restoration. 


\section{Introducción}

Los acontecimientos de desastres naturales de acción natural y antrópica, ocurridos durante los últimos diez años en Colombia y la reciente temporada de verano, evidencian la probabilidad de ocurrencia de eventos indeseables en aumento tales como los incendios forestales e inclusive las quemas prohibidas, que pueden representar en la realidad una situación de peligro en el incremento de las tipologías de riesgos, como consecuencia de las debilidades logísticas en materia de prevención, ante la amenaza de la materialización del riesgo; además de exponer la estrecha vinculación entre los deberes y obligaciones del Estado colombiano, para el caso, en virtud del Artículo 8 de la Constitución Política de Colombia de 1991: es obligación del Estado y de las personas proteger las riquezas culturales y naturales de la Nación (...) como garante de un derecho constitucional, calidad de vida y como aporte al proceso de paz.

Aunque en la primera de las normas se habla de proteger las riquezas culturales y naturales de la Nación y en la segunda de proteger los recursos culturales y naturales del país, se debe entender que se trata del mismo bien jurídico (Amaya, O. 2002).

En plena sequia años 2015 y 2016, sobresale por sus efectos el fenómeno del niño pero en esencia, el método utilizado para agotar los incendios forestales que se apoya en elementos primarios o quizás básicos como: baldes, recatones y picas, tal como acontecía en la época medioeval. El método sugerido combinado para detectar y combatir oportunamente incendios forestales consiste en la implementación efectiva de tecnologías aeronáuticas, como lo es, el sistema de aviones especializados Air Tractor específicamente el AT-802F una aeronave de buena maniobrabilidad, económica y de efectividad operacional, que ejecuta una singular capacidad de vertimiento de agua, también en combinación con productos retardantes, limitando el poder devastador de los incendios forestales; ya que está diseñado en su totalidad para estas operaciones. El AT-802F utiliza una compuerta computarizada de avanzada para controlar los niveles de cobertura con exactitud "extensión del incendio y área a cubrir" dada la topografía abrupta y compleja de nuestro país beneficia los análisis técnicos de sistemas de protección en sistemas forestales del orden nacional.

En este orden de ideas, no cabe duda que los incendios forestales que estallan en las montañas afectan el ecosistema forestal colocando en riesgo la protección, conservación y regularización del mismo.

\section{Método}

El presente artículo ofrece el desarrollo de la siguiente metodología. En primer lugar, se realiza un análisis de carácter cuantitativo de los dos últimos años específicamente 2015 y 2016, evidenciando el compromiso de la estructura vegetal en dos vertientes: reforestación y restauración, teniendo como indicador las hectáreas por año pérdidas. En segundo lugar, se expondrá el caso Chile, en donde la ocurrencia de eventos forestales en los andes chilenos, presentó una reducción de $42 \%$ luego de la implementación de este sistema de aviones especializados Air Tractor. Finalmente, se profundiza en la implementación sugerida de tecnología aeronáutica para controlar incendios forestales, suministrando las respectivas especificaciones técnicas de la aeronave Air Tractor AT-802F y sus condiciones de maniobrabilidad.

\section{Análisis cuantitativo}

En el análisis cuantitativo en territorio nacional de los dos últimos años 2015 - 2016 se reconoce que la incorporación de la gestión del riesgo de desastres específicamente los incendios forestales ocurridos y evidenciados en determinados casos de origen natural y antrópico, promueven la ocurrencia de eventos forestales de manera significativa, contribuyendo así al crecimiento del riesgo ${ }^{5}$ debido a la escasa disponibilidad de recursos de las entidades descentralizadas y en los órganos competentes de las entidades territoriales, destinados a la prevención y atención de desastres y calamidades, además, de una ausente conciencia forestal. Como se observa, en el consolidado de afectación en las Figuras 1 y 2, los eventos registrados inciden en un efecto de interacción entre las áreas afectadas y las zonas de ubicación (topografía compleja), puesto que la interacción es significativamente mayor en zonas montañosas y menor en zonas de llanura, lo que evidencia una relación entre ambas variables, condicionada en la capacidad de reacción a la zona del siniestro por un ataque inicial efectivo que reduzca la superficie afectada. El método de aplicación no refleja la contundencia requerida, de ahí la derivada de las áreas afectadas y su impacto en el medio ambiente, bajo el condicionamiento de medición en el impacto del nivel de reforestación y restauración ${ }^{6}$.

\footnotetext{
${ }^{5}$ Riesgo ambiental se describe como la probabilidad de que ocurra un efecto adverso a nivel individual o poblacional, por la exposición a concentraciones elevadas o por dosis específicas de un agente ambiental peligroso (Vallejo, M. \& Baena, C., 2007).

${ }^{6}$ La restauración áreas quemadas, o restauración ecológica post-fuego, es el proceso de ayudar en la recuperación de un ecosistema que han sido degradado, dañado o destruido por el fuego
} 


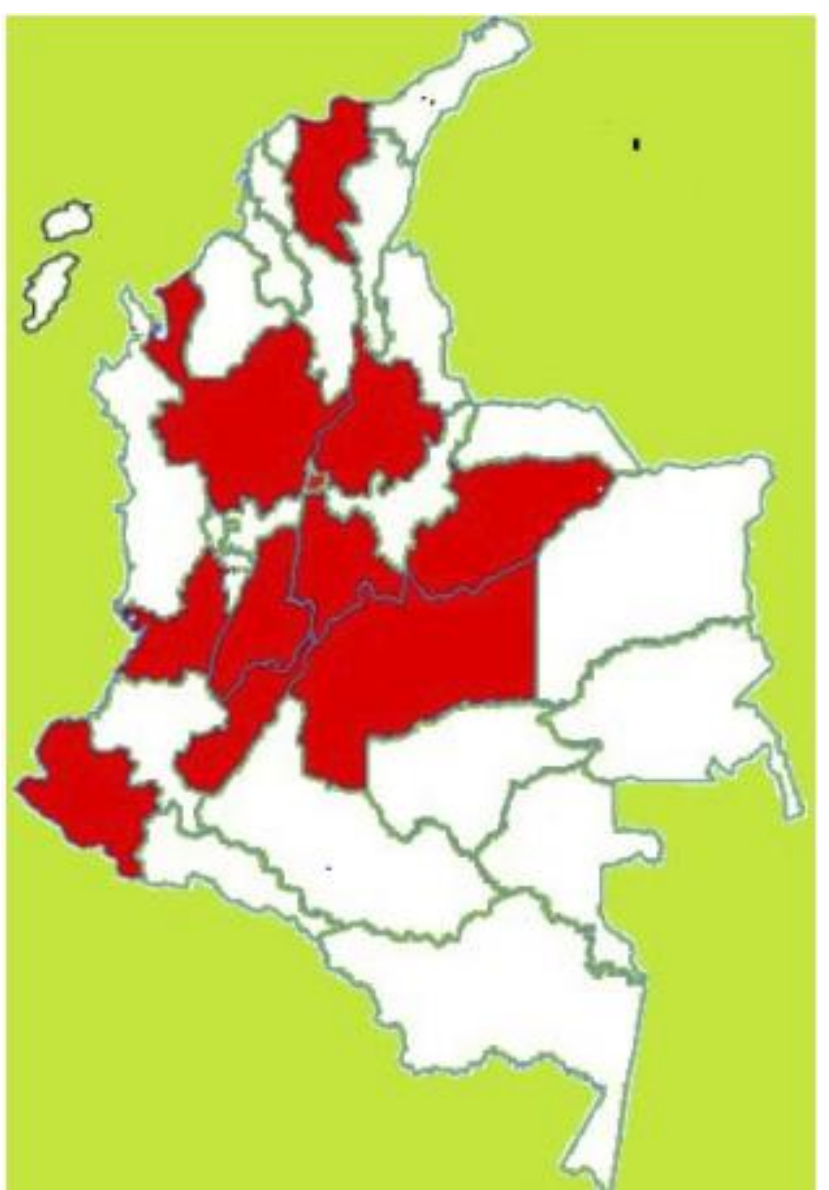

\begin{tabular}{cc}
\hline Departamento & Evento \\
\hline Cundinamarca & 27 \\
\hline Huila & 6 \\
\hline Tolima & 4 \\
\hline Valle del Cauca & 3 \\
\hline Antioquia & 1 \\
\hline Casanare & 1 \\
\hline Magdalena & 1 \\
\hline Meta & 1 \\
\hline Nariño & 1 \\
\hline Santander & 1
\end{tabular}

Figura 1. Afectación por incendios forestales consolidado año 2015.

Fuente: Dirección Nacional Bomberos Colombia. [En línea] Disponible en: http://www. http://bomberos.mininterior.gov.co/

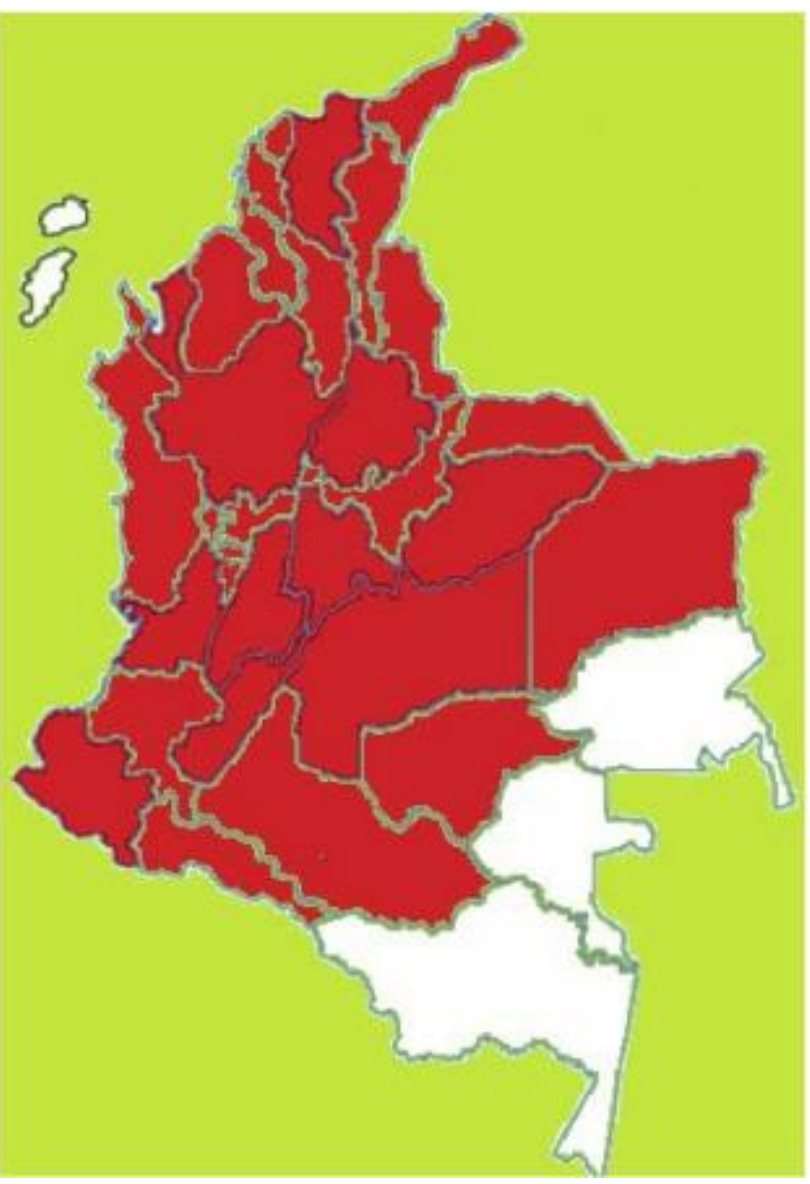

\begin{tabular}{lc}
\hline Cundinamarca & 643 \\
\hline Antioquia & 400 \\
\hline Casanare & 195 \\
\hline Meta & 164 \\
\hline Risaralda & 160 \\
\hline Valle del Cauca & 137 \\
\hline Boyacá & 98 \\
\hline Magdalena & 93 \\
\hline Santander & 84 \\
\hline Caldas & 61 \\
\hline La Guajira & 50 \\
\hline Tolima & 49 \\
\hline Guaviare & 47 \\
\hline Huila & 45 \\
\hline Cauca & 35 \\
\hline
\end{tabular}

\begin{tabular}{lc}
\hline Cesar & 32 \\
\hline Arauca & 28 \\
\hline Sucre & 22 \\
\hline $\begin{array}{l}\text { Norte de } \\
\text { Santander }\end{array}$ & 21 \\
\hline Nariño & 12 \\
\hline Caquetá & 6 \\
\hline Córdoba & 6 \\
\hline Bolívar & 4 \\
\hline Choco & 3 \\
\hline Putumayo & 3 \\
\hline Atlántico & 2 \\
\hline Quindío & 1 \\
\hline Vichada & 1 \\
\hline
\end{tabular}

Figura 2. Afectación por incendios forestales del 1 de enero al 14 de julio de 2016.

Fuente: Dirección Nacional Bomberos Colombia. [En línea] Disponible en: http://bomberos.mininterior.gov.co/ 
Los cambios drásticos de las variables influyen en el conocimiento del riesgo y en el manejo adecuado de desastres que deben contemplar en su complementariedad una prevención y mitigación del riesgo desde la planificación (partes de un incendio forestal). Es conveniente aclarar que la gestión del riesgo existente no se debe limitar a la zona geográfica donde se materializa la emergencia, sino que implica la evaluación integral de los espacios urbanos y rurales en una unificación y simplificación regional coordinada partiendo del ámbito municipal, a la gobernabilidad del riesgo comenzando con la perspectiva de lo público en una subyacente política del riesgo evaluada (en tres fases: seguimiento, monitoreo y control) anexo a la actualización del sistema de mitigación aéreo adjunto a los métodos convencionales, en la masiva, de una adecuada y eficiente aplicación a los niveles de ocurrencia.

Tabla 1.

Perfil aéreo de apoyo.

Afectación por incendios forestales vs hectáreas afectadas

\begin{tabular}{ccccc}
\hline Año & Eventos & $\begin{array}{c}\text { Hectáreas } \\
\text { afectadas }\end{array}$ & \multicolumn{2}{c}{ Apoyos aéreos } \\
\hline & & Efectivos & No efectivos \\
\hline 2015 & 4.617 & $120.041,07$ & $* *$ & $* *$ \\
\hline $2016^{*}$ & 2.403 & $107.626,90$ & 20 & 40 \\
\hline & $\sum 227.667,97$ & & $\Sigma$ & 60 \\
\hline
\end{tabular}

Fuente: Elaboración propia. Los reportes de incendios forestales pueden consultarse en el siguiente enlace: http://bomberos.mininterior.gov.co/

*Periodo comprendido del 1 de enero al 14 de julio de 2016, **No reportado.

En la Tabla 1 se han registrado las hectáreas afectadas como consecuencia de los eventos ocurridos, teniendo en cuenta los apoyos aéreos (adaptados no específicos para este tipo de operaciones) realizados que en la sumatoria total excede el $66 \%$ de No efectividad, dado el alto nivel de afectación de la capa terrestre, que compromete significativamente el patrimonio natural y cultural de la nación.

En este sentido, investigaciones científicas encaminadas a la protección y conservación del medio ambiente sostienen siempre que el desarrollo forestal está cada vez más en riesgo, cabe aclarar que el ámbito normativo en su estructura es limitado y no es determinante en la restauración ecológica. Teniendo en cuenta como Greunal (2012) lo refiere "El éxito en la restauración también dependerá de los costos, las fuentes de financiamiento, la voluntad política de las instituciones interesadas en la restauración; pero ante todo de la colaboración y participación de las comunidades locales en los proyectos" (p. 9). Restaurar un ecosistema total o parcialmente afectado, considera devol- ver en el tiempo su estructura, composición, diversidad de especies (flora y fauna) y funcionamiento de la manera más cercana a su estado inicial, trabajando sobre la sucesión secundaria. La restauración ecológica post-fuego toma importancia por lo difícil de su aplicación, la mayoría de las experiencias en el mundo considera sólo una parte de los objetivos de la restauración ecológica, focalizándose en contrarrestar el desbalance hídrico originado después de un incendio es decir restauración hidrológica y su impacto en las poblaciones aguas abajo de la cuenca afectada como la Corporación Nacional Forestal de Chile en adelante Conaf lo refiere. Lo que no permite una optimización simultanea determinada por los niveles de restauración que proporcione; una solución exitosa y de funcionabilidad en condiciones semejantes a las presentadas originalmente. Para el caso colombiano, las hectáreas afectadas presentan un dato que debe ser manejado convenientemente por el problema que generan para encontrar otro que se busca "reforestación".

\section{Caso Chile}

Como se puede evidenciar en la Figura 3, el nivel de ocurrencia de los incendios forestales ha ido en incremento, de acuerdo a sólidas investigaciones realizadas por la Conaf de Chile, en la mayoría de los casos, las situaciones de riesgo inminente han sido propiciadas por acciones antrópicas derivadas de conductas delictivas. Es importante observar que el índice de daño de los incendios forestales se ha elevado considerablemente en el último decenio, presentando su pico más alto en el año 2014, para el año 2015 la materialización del riesgo en los andes chilenos, presentó una reducción de $42 \%$, luego de la implementación del Sistema Aéreo Air Tracto AT-802F, como el mejor método para agotar incendios forestales, hasta el momento, en en-

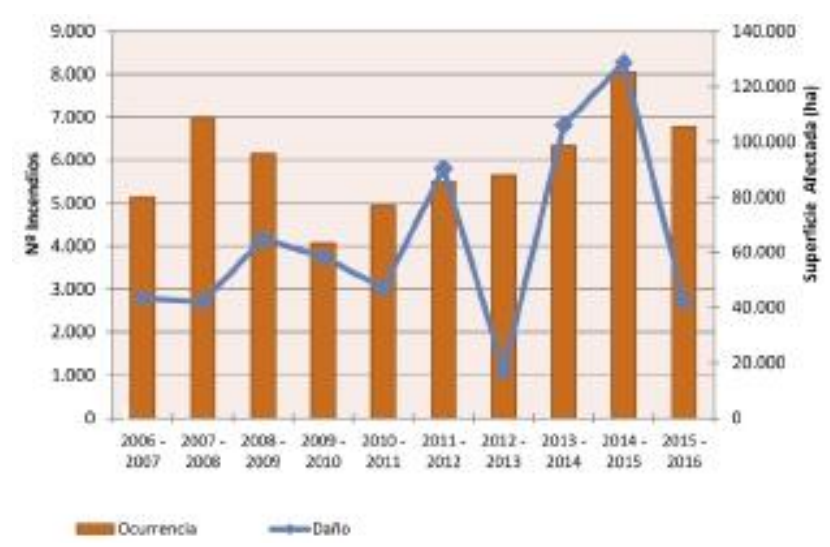

Figura 3. Ocurrencia y Afectación por incendios forestales decenio 2007-2016.

Fuente: Estadísticas. [En línea] Disponible en: http://www.conaf.cl/ incendios-forestales/incendios-forestales-en-chile/ 
tornos geográficos de difícil acceso (topografía abrupta). Además, de reducir el compromiso de vidas humanas y de áreas afectadas.

Es de aclarar que la implementación de este sistema aéreo se apoyó en la identificación de variables relevantes necesitadas de control y soportadas en amplios estudios técnicos de análisis poblacional en entornos rurales principalmente inducidos a la prevención de incendios forestales, para construir el modelo de los fenómenos de reforestación y restauración a estudiar (Ortega, M. 2005).

\section{Implementación de tecnología aeronáutica}

La incidencia reportada de eventos cuenta con unas 227.667,97 hectáreas afectadas en el territorio nacional, lo que evidencia una elevada medición que infiere del tratamiento previo e in situ a la contingencia, con probabilidad gradual y progresiva al aumento. Experiencias previas plantean la necesidad de implementar un sistema aéreo que permita ajustar la naturaleza de estos eventos, como el sistema de aviones especializados Air Tractor específicamente el AT-802F de última generación (ver Figura 4). Esta aeronave presenta una buena maniobrabilidad, es económica y de gran efectividad operacional, esta tecnología aeronáutica presenta ventajas de velocidad considerables, lo que podría aumentar la capacidad de reacción a la zona del siniestro, en lo que terminológicamente se denomina ataque inicial (atendiendo las variables de generación radiación, conducción y/o fricción), reflejado en la reducción de la superficie al control total del incendio y permitiendo a su vez una cobertura nacional que reduzca la ocurrencia de incendios forestales.

Su capacidad de vertimiento es de 3000 a 3100 litros aproximadamente dependiendo de las condiciones de altitud y temperatura, su costo en orden de magnitud oscila entre 1.700 .000 a 1.900 .000 US aproximadamente. Para una mayor ilustración las especificaciones técnicas del fabricante se encuentran en las Tablas 2 y 3.

Cabe aclarar que todo sistema especializado para el caso tecnología aeronáutica, requiere pilotos calificados y certificados para este tipo de tareas. Es decir, no son aeronaves civiles o militares "adaptadas" para este tipo de eventos, sino que por el contrario el Air Tractor AT-802F, es una aeronave de última generación, específicamente diseñada en su totalidad para apagar incendios forestales.

En este sentido, es importante señalar que adjunto a una eficiente mitigación del riesgo se deben desarrollar correctos análisis técnicos del sistema topográfico e inclusive de la variabilidad climática e investigaciones confiables a la población afectada que permitan sociabilizar la gestión del riesgo, principalmente en la asimilación de medidas de prevención que generen una conciencia forestal en Colombia y un compromiso fuerte por parte del Ministerio Público (Banco Mundial, 2012).

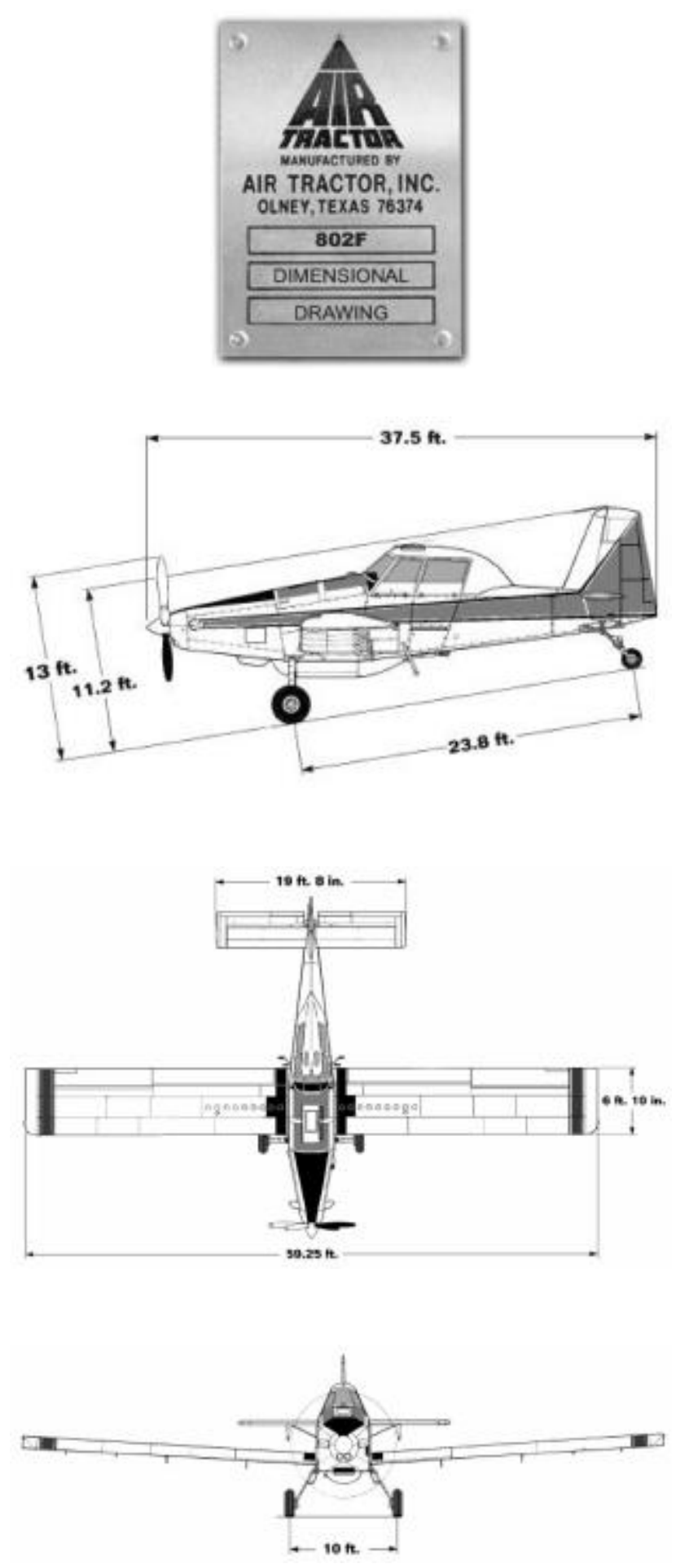

Figura 4. Diseño aeronave AT-802F.

Fuente: Air Tractor. [En línea] Disponible en: http://www.airtractor. com/es/aircraft/802f 
CIENCIA Y PODER AÉREO | Revista Científica de la Escuela de Postgrados de la Fuerza Aérea Colombiana | Vol. 11 | Enero - Diciembre de 2016

Tabla 2.

Especificaciones técnicas aeronave AT-802F.

\begin{tabular}{llllll}
\hline Engine Type & P\&W PT6A-67AG & $\begin{array}{l}\text { Empty Wt. } \\
\text { w/Spray Equip.: }\end{array}$ & 7,210 lbs. (3 270 kg) & Wing Span & 59.2 ft. (18,04 m) \\
\hline Engine S.H.P.: & 1,350 @ 1,700 RPM & Useful Load: & 8,790 lbs. (3 987 kg) & Wing Area: & 401 sq. ft. (37,29 m2) \\
\hline Propeller: & $\begin{array}{l}\text { Hartzell HC-B5MA-3D/ } \\
\text { M11691NS }\end{array}$ & Hopper Capacity: & 820 U.S. gal. (3 104 L) & Main Wheel Size: & 32.0 in. \\
\hline Take-Off Weight: & 16,000 lbs. (7 257 kg) & Fuel Capacity: & 254 U.S. gal. (961 L) & Tail Wheel Size: & $6.25 \times 6$ \\
\hline Landing Weight: & $16,000$ lbs. (7 $257 \mathrm{~kg})$ & & & & Specifications \\
\hline
\end{tabular}

Fuente: Air Tractor. [En línea] Disponible en: http://www.airtractor.com/es/aircraft/802f

Tabla 3.

Performance aeronave AT-802F.

\begin{tabular}{l|lllll}
\hline Cruise Speed: & $221 \mathrm{mph}(356 \mathrm{kph})$ & $\begin{array}{l}\text { Stall Speed, } \\
\text { Flaps Up: }\end{array}$ & $\begin{array}{l}105 \mathrm{mph}(169 \mathrm{kph}) \text { at } \\
16,000 \mathrm{lbs}(7257 \mathrm{~kg})\end{array}$ & Rate of Climb: & $\begin{array}{l}850 \mathrm{fpm}(259 \mathrm{mpm}) \text { at } \\
16,000 \mathrm{lbs}(7257 \mathrm{~kg})\end{array}$ \\
\hline $\begin{array}{l}\text { Working Speed } \\
\text { (Typical): }\end{array}$ & $\begin{array}{l}120-125 \mathrm{mph}(193- \\
201 \mathrm{kph})\end{array}$ & $\begin{array}{l}\text { Stall Speed, } \\
\text { Flaps Down }\end{array}$ & $\begin{array}{l}91 \mathrm{mph}(146 \mathrm{kph}) \text { at } \\
16,000 \mathrm{lbs}(7257 \mathrm{~kg})\end{array}$ & Take-Off Distance: & $\begin{array}{l}2,000 \mathrm{ft}(610 \mathrm{~m}) \text { at } \\
16,000 \mathrm{lbs}(7257 \mathrm{~kg})\end{array}$ \\
\hline $\begin{array}{l}\text { Range, Economy } \\
\text { Cruise at 8,000 ft: }\end{array}$ & $800 \mathrm{mi}(1287 \mathrm{~km})$ & $\begin{array}{l}\text { Stall Speed } \\
\text { as Usually } \\
\text { Landed: }\end{array}$ & $69 \mathrm{mph}(111 \mathrm{kph})$ & Performance \\
\hline
\end{tabular}

Fuente: Air Tractor. [En línea] Disponible en: http://www.airtractor.com/es/aircraft/802f

Esta situación en particular puede definirse como la producción de un fenómeno de frecuencia considerable que debe ser sometido a un estudio de observancia constante. En directa proporción la aplicación del método sugerido combinado como control de aplicación supone una tarea de estricta vigilancia que en las condiciones previstas elimina la incertidumbre porque admite previsión y confiabilidad en el procedimiento utilizado.

\section{Conclusiones}

El nivel de efectividad en la mitigación del riesgo está dado en el ámbito de aplicabilidad de los sistemas tecnológicos que tiene directa relación con el costo de la operación y la capacidad de respuesta como lo indica este artículo al recomendar el sistema de aviones especializados Air Tractor específicamente el AT-802F de última generación; por lo que deben integrarse los métodos más eficaces y eficientes en la actualidad, que signifiquen la disminución porcentual del riesgo actual y permanente, dando verdadero cumplimiento a lo descrito en la Constitución Ecológica de Colombia.

Resulta urgente la utilización adecuada de estos elementos que tendrá impacto en la medida que su ejecución considere la educación y formación requeridas de manera cualificada, sin poner en riesgo la seguridad nacional en el caso de situaciones críticas, además de todas aquellas que las entidades implicadas sobre todo el Ministerio Público, consideren relevantes.

El trabajo diligente permitirá alcanzar una existencia fácil en el futuro.

Lao Tsé

Tao Te Ching

\section{Referencias}

Air Tractor. [En línea] Disponible en http://www.airtractor.com/es/ aircraft/802f, recuperado el 20 de julio de 2016.

Alegría, M.; Álvarez G., et al., (2007). Incorporación de los principios de la gestión integrada de recursos hídricos en los marcos legales en América Latina: experiencias aprendidas. Universidad Externado de Colombia.

Amaya, O. (2002). La constitución ecológica de Colombia. Universidad Externado de Colombia

Amaya, O; Castiblanco C., et al., (2007). Control fiscal ambiental. Universidad Externado de Colombia. 
Banco Mundial Colombia, (2012). Análisis de la gestión del riesgo de desastres en Colombia. Ed. Banco Mundial.

Constitución Política de Colombia, (2009). Bicentenario de la Independencia de Colombia 1810 2010. Senado de la República. Imprenta Nacional de Colombia,2.a.ed.

Dirección Nacional de Bomberos de Colombia. (2016). [En línea] Disponible en: http://bomberos.mininterior.gov.co/, recuperado el 11 de julio de 2016.

Greunal. (2010). Guías Técnicas para la Restauración Ecológica de Ecosistemas. Colombia: Universidad Nacional de Colombia.

Ortega, M. (2005). Programa educación para la prevención del riesgo de incendios forestales a través de las TICS. Chile: Corporación Nacional Forestal.

Rojas, C. (2004). Evolución de las características y de los principios del derecho internacional ambiental y su aplicación en Colombia. Colombia: Universidad Externado de Colombia.

Taylor, S.; \& Bogdan, R. (1996). Introducción a los métodos cualitativos de investigación. Barcelona: Ediciones Paidós Ibérica S.A. $3^{\circ}$ Reimpresión.

Vallejo, M.; \& Baena, C. (2007). Toxicología ambiental. Colombia: Ed. Grupo Empresarial Wills Ltda.

Vladimir, A. (2008). Tao Te Ching. Printed by Lulu. Canada. 